\title{
Sizes of the actively aerated zone and methods of the jet aerator calculation
}

\author{
Malokhat Abdukodyrova ${ }^{1}$, Maria Radkevich $^{1 *}$, Kamila Shipilova ${ }^{1}$ and Abdusamin Gapirov ${ }^{2}$ \\ ${ }^{1}$ Tashkent Institute of Irrigation and Agricultural Mechanization Engineers, Tashkent, Uzbekistan \\ ${ }^{2}$ Tashkent State Transport University, Tashkent, Uzbekistan
}

\begin{abstract}
The possibilities of using jet aeration for biological wastewater treatment in small facilities were considered in the article. In shallow depth reservoirs, studies were carried out to change the sizes of the actively aerated zone with a decrease in the height of the liquid layer in the reservoir. The research was conducted for jet aerators with nozzles of the "keyway" type holes. It was established that a decrease in the relative depth of the reservoir makes it possible to increase the share of the beneficial use of the reservoir volume. This suggests that jet aeration will be most effective for reservoirs of relatively shallow depth. Based on the research results, a method for calculating a jet aerator was proposed, which makes it possible to evaluate the necessary parameters of the aerator based on the determination of the volumetric mass transfer coefficient.
\end{abstract}

\section{Introduction}

The Republic of Uzbekistan is a country with a significantly developed agriculture and sizeable industrial production. However, in recent years, there has been active development of small industrial enterprises; this development leads to the emergence of small settlements and urban-type settlements with their infrastructure, particularly with small treatment facilities for one or several settlements. Wastewater is formed, as a rule, due to car washing from minor repair sites and household wastewater. In addition, wastewater from the canning shops or tanneries can enter the treatment facility.

Such a runoff contains easily oxidized organic substances that are part of the household runoff and difficultly oxidized organic substances in hydrocarbons of various fuels and lubricants. The volumes of wastewater from individual enterprises are small. In contrast, the treatment systems capable of bringing the quality of the treated wastewater to the norms of discharge into the sewer are usually complex and require qualified service personnel. In this regard, such runoffs are discharged without treatment into the existing sewer network [1]. Therefore, sewage treatment plants for small settlements in rural areas receiving mixed effluents of a complex composition should be simple to design and operate, allowing the purification of treated wastewater required for the discharge into open water bodies.

\footnotetext{
*Corresponding author: m.radkevich@tiiame.uz
} 
Basically, biological ponds and small treatment plants of biological purification using wetland plants [2] are arranged to treat the listed wastewaters; however, to intensify the treatment, it is desirable to provide artificial aeration. Aeration, as a method of saturating reservoirs with air oxygen, aimed to maintain the required water quality, is widely used in the practice of biological wastewater treatment $[3,4,5,6]$. Considering the relatively lowincome level in Uzbekistan according to the international rating, it is necessary to develop relatively inexpensive wastewater aeration processes.

Considering the difficulties associated with the operation of aerators of pneumatic and mechanical mixing, many researchers turned to the study and design of devices with jet mixing $[3,7,8,9,10,11]$. Of all the known methods, jet gas-liquid aeration is the most effective way to oxygenate water [8]. This method allows to generate air bubbles and distribute them in the volume of water and control the parameters of aeration, namely, the size of the formed bubbles and their distribution in the treated volume.

The mechanism of a liquid saturation with gas in such devices is based on the injection of a gas by a jet of a falling liquid flowing out of a nozzle. After leaving the nozzle, the surface of the liquid jet, moving in the gaseous medium, becomes "rough". The gas penetrates the depressions of "roughness" and is carried away by jet into co-motion [10]. Then, the jet penetrates deep into the reaction volume, where the gas is dispersed in the form of small bubbles, forming a gas-liquid mixture with a developed interphase surface in the contact zone.

The performance of aerators is significantly influenced by the shape and size of the nozzle. The study of this influence is devoted to the works of many researchers [13 - 18].

Ledyan Y. et al. (1986) [17] found that an increase in the transverse dimensions of the jet at the same flow rates leads to a sharp decrease in the degree of injection (for example, with an increase in the diameter of a round jet by 1.6 times, the degree of injection decreases by 4.67 times).

Ledyan Y. (2008) [18] investigated the aerating capacity of jets of circular and annular cross-sections with an outer diameter from 5 to $9 \mathrm{~mm}$ and an inner diameter from 4 to 8 $\mathrm{mm}$ (for jets of an annular cross-section). It was stated that for jets of circular cross-section, the degree of injection increases with decreasing diameter. For jets of annular cross-section, the degree of injection increases with decreasing thickness of the jet wall. The authors claim that annular jets provide a significantly higher degree of ejection. However, adjusting the jet wall thickness for jets of annular cross-section complicates the operation of such devices.

Meshcheryakov et al. [17] conducted a series of experiments to determine the injecting ability of a jet of circular and flat rectangular cross-sections (with equal cross-sectional areas) nozzles), which showed that the jet shape is one of the factors determining its injecting ability. Thus, for a jet of a flat rectangular section with a fluid flow rate of $2.1 \mathrm{l} / \mathrm{s}$, the injection capacity was $66 \%$ higher than for a jet of a circular section. However, the article does not explain the reasons for the higher injection capacity of jets of a flat crosssection.

Kusabiraki [18], investigating the characteristics of gas capture by descending vertical liquid jets, also found that the difference in the amount of captured gas is related to the difference in the jet shape before it penetrates the free surface of the liquid.

According to Van de Sand [19], the configuration of the nozzle is of great importance in assessing the injection ability of the jet. Conducting experimental studies with nozzles of various configurations, he concluded that the nozzle, which has an elongated section with parallel walls, has the maximum aerating ability. The liquid flowing through such a nozzle ensures the turbulization of the jet before it leaves the nozzle, which can lead to its higher injecting capacity. Abaev, Safronova, and others came to the same conclusion [20, 21, 22]. 
The authors of this article conducted studies with nozzles having a different number of holes with the same total area of the holes.

Studies devoted to the injection ability of freely falling jets are described in [10, 11, 23]. The authors of these studies concluded that a jet with a higher turbulence intensity at a shorter length injects a more significant amount of gas due to early ruptures on the jet surface, contributing to a more developed 'roughness'. The jet turbulence, in their opinion, is directly related to the configuration of the nozzle.

Numerous data obtained by other researchers [7, 9, 10, 17], and the authors' observations, show that in a gas-saturated volume of a liquid, it is possible to distinguish the so-called actively aerated zone, permeated with gas bubbles, and the surrounding homogeneous zone. Determining the size of the active zone is necessary to obtain design equations. The main dimension of the actively aerated zone should be considered the depth of the jet penetration $h_{\text {pen }}$.

To determine the depth of jet penetration, several formulas were proposed, for example:

$$
\begin{aligned}
& h_{\text {pen }}=10 U_{n} d_{n} \\
& h_{p e n}=2.6\left(U_{n} d_{n}\right)^{0.7}
\end{aligned}
$$

where $U_{n}$ is the jet velocity in the nozzle, $\mathrm{m} / \mathrm{s}, d_{n}$ is the nozzle diameter, $\mathrm{m}$.

The authors of $[23,24]$ believe that the actively aerated (gas-liquid plume) zone has a cylindrical shape, and propose to determine its diameter by the following formula:

$$
d_{p l}=0.44 h_{p e n}
$$

Lobov [10] attempted to determine the shape and size of the actively aerated zone using the equation of motion of a gas bubble in a liquid flow. However, the resulting equation turned out to be cumbersome and lacking an analytical solution. The numerical solution of this equation was performed for the initial jet velocity $U_{n}=10 \mathrm{~m} / \mathrm{s}$ and the nozzle hole diameter $\mathrm{d}_{\mathrm{n}}=12 \mathrm{~mm}$. According to this equation, the actively aerated zone shape approaches a paraboloid.

In [25], the motion of a single bubble in a submerged jet was visualized using a digital camera. The rather complex trajectory of the bubble can hardly characterize the size of the actively aerated zone since it includes not one but many bubbles.

Based on the data considered, it can be concluded that, despite numerous studies, there is no consensus on the size of the actively aerated zone. However, this issue remains relevant when trying to calculate the jet aerator.

The limiting factor in the wider use of jet-type devices is the imperfection of the structures and the absence of a scientifically substantiated method for calculating these aerators. This study aims to develop a method for calculating jet devices based on theoretical and experimental studies.

\section{Materials and Methods}

The purpose of the experiments was to determine the optimal height of the aerated liquid layer with the maximum possible volume of the reservoir.

\subsection{Materials}

An experimental setup was used for the study; it includes a reservoir with a scale for measuring the liquid level and a weir, a supply pipeline with a head-pressure control device, and replaceable nozzles with holes in the form of a keyway (Table 1). 
Table 1. Parameters of nozzles

\begin{tabular}{|c|c|c|c|c|c|}
\hline Type & $\begin{array}{c}\text { Number of } \\
\text { holes }\end{array}$ & \multicolumn{2}{|c|}{$\begin{array}{c}\text { Dimensions of holes, } \\
\mathrm{mm}\end{array}$} & $\begin{array}{c}\text { Total area of } \\
\text { holes S, } \mathrm{mm}^{2}\end{array}$ & $\begin{array}{c}\text { Total perimeter } \\
\text { of holes P, mm }\end{array}$ \\
\cline { 3 - 4 } & & $\mathrm{L}$ & $\mathrm{b}=\mathrm{d}$ & & 104 \\
\hline I & 1 & 29.2 & 14.6 & 595 & 147 \\
\hline II & 2 & 20.6 & 10.3 & 595 & 208.5 \\
\hline III & 4 & 14.6 & 7.3 & 595 & 297 \\
\hline IV & 8 & 10.4 & 5.2 & 595 & \multicolumn{2}{c}{} \\
\hline
\end{tabular}

To fix the size of the actively aerated zone, a Canon EOS M6 Mark II digital camera with a shooting rate of 14 frames per second was used.

\subsection{Experimental technique}

The experiments consisted of photographing the process of penetration of the gas-liquid jet into the thickness of the aerated liquid. The jet length in the air was $30 \mathrm{~cm}$, and the reservoir width was 1.06 of the jet penetration depth.

The liquid level changed sequentially. Initially, the liquid level was selected so that the gas-liquid jet was completely immersed without touching the bottom. Then the liquid level decreased, and the jet broke against the bottom of the reservoir, and air bubbles were distributed over the most significant part of the volume.

For the experiment, the ratio between the thickness of the liquid layer hp and the depth of jet penetration $h_{p e n}$ was chosen as the main factor affecting the size of the actively aerated zone. The experiment was conducted at the following levels of this factor

\begin{tabular}{|l|l|l|l|l|l|}
\hline $\mathrm{h}_{\mathrm{res}} / \mathrm{h}_{\text {pen }}$ & 1.05 & 1 & 0.7 & 0.6 & 0.5 \\
\hline
\end{tabular}

The number of measurements at each level is the same and equals $\mathrm{N}=10$. To process the experimental data and to test the null hypothesis on the substantial influence of the selected factor on the size of the actively aerated zone, a single-factor analysis of variance (a oneway ANOVA test) was performed.

\section{Results and discussion}

Analysis of variance of the experimental results made it possible to establish the degree of influence of the relative height of the liquid layer in the reservoir $h_{t} / h_{\text {pen }}$ on the sizes of the actively aerated zone (the fraction of the reservoir volume in \%, occupied by the spray pattern of gas bubbles). Analysis of variance results are presented in Table 2.

Table 2. Results of analysis of variance

\begin{tabular}{|c|c|c|c|c|}
\hline $\begin{array}{c}\text { Total mean } \\
\bar{x}, \%\end{array}$ & $\begin{array}{c}\text { Factorial } \\
\text { variance } s_{f}^{2}\end{array}$ & $\begin{array}{c}\text { Residual } \\
\text { variance } s_{o}^{2}\end{array}$ & $\begin{array}{c}\text { Fisher's variance } \\
\text { ratio }_{\text {obser }}\end{array}$ & $\begin{array}{c}\text { Tabular value of Fisher's } \\
\text { variance ratio } \mathrm{f}_{\text {tab }}\end{array}$ \\
\hline 43.19 & 769.68 & 0.0972 & 7918.52 & 2.53 \\
\hline
\end{tabular}

Since $f_{\text {obser }}>f_{\text {tab }}$, the group means differ significantly. Consequently, we accept the null hypothesis about the significant effect of the height of the liquid layer relative to the jet penetration depth on the size of the actively aerated zone (and we reject the null hypothesis about the equality of group means).

Experimental photographs were used to determine the sizes of the actively aerated zone for various ratios of the reservoir depth (the height of the liquid layer) and the jet penetration depth (Fig. 1). The dimensions in the figures are in relative units. 


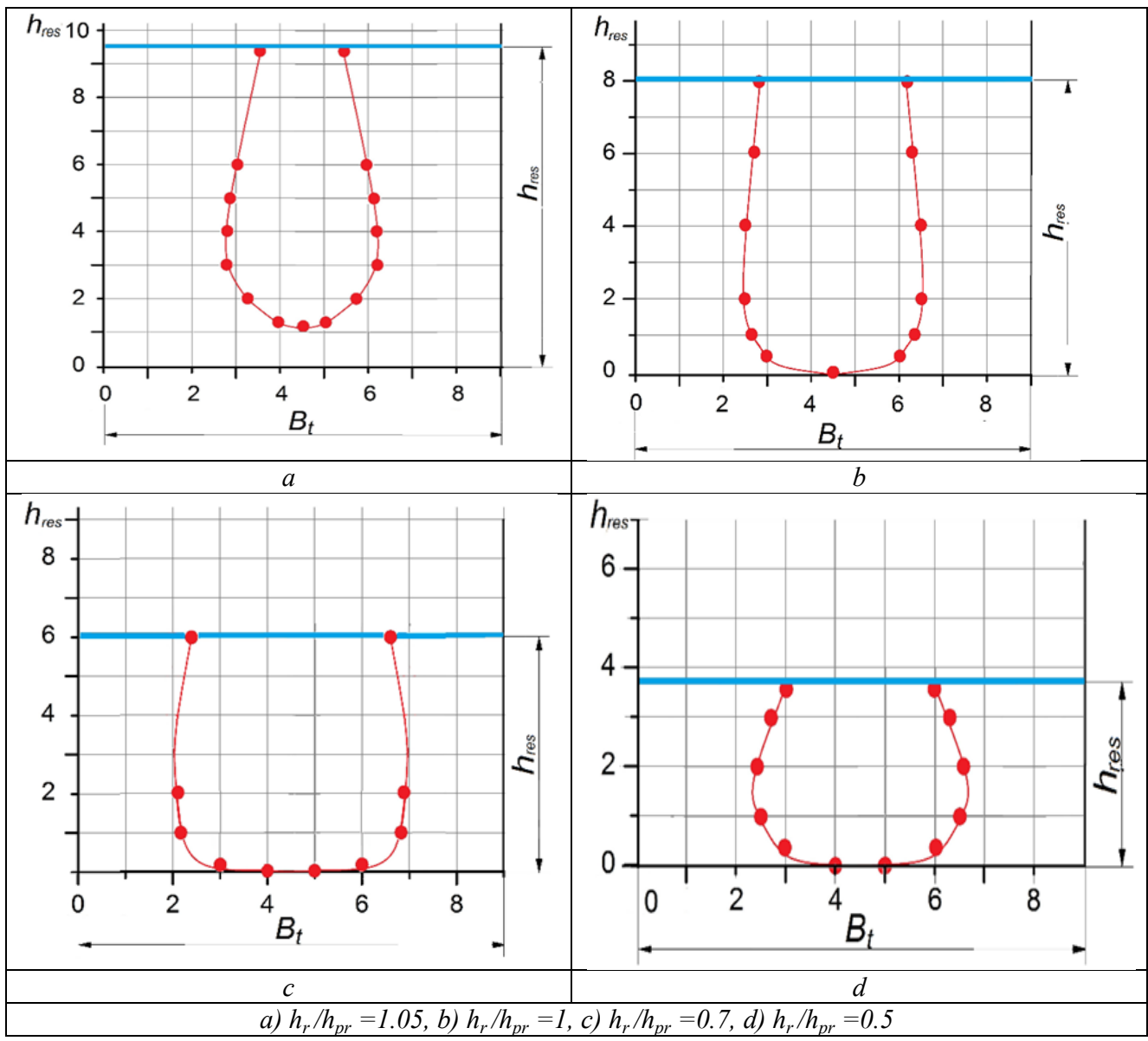

Fig.1. Shape of the aerated zone at different thickness of water layer in the reservoir $\left(\mathrm{B}_{\mathrm{t}}-\operatorname{tank}\right.$ width, $h_{\text {res }}$ - reservoir depth (liquid layer))

As seen from the graphs, a freely forming gas-liquid plume, when it fully immersed in a liquid (without depth restrictions), acquires a drop-like shape (Fig. 1, a), but with a decrease in the height of the water layer, it gradually approaches the shape of a cylinder (Figs. 1, b, c), and then the shape of a flattened drop (Fig. 1,d). The data obtained are consistent with other researchers [9] on the drop-like shape of a freely forming jet spray pattern. Considering that other authors have not studied the formation of a jet spray pattern when the height of the water layer is limited, there is no way to compare the other data we obtained.

Experiments conducted with jets with different characteristics (velocity, flow rate, nozzle shape) made it possible to construct a generalized graph of dependence of the utilization factor of reservoir volume (with width $1.06 h_{\text {pen }}$ ) on the height of the liquid level, expressed in fractions of the total (possible) depth of penetration of the liquid jet (Fig. 2). 


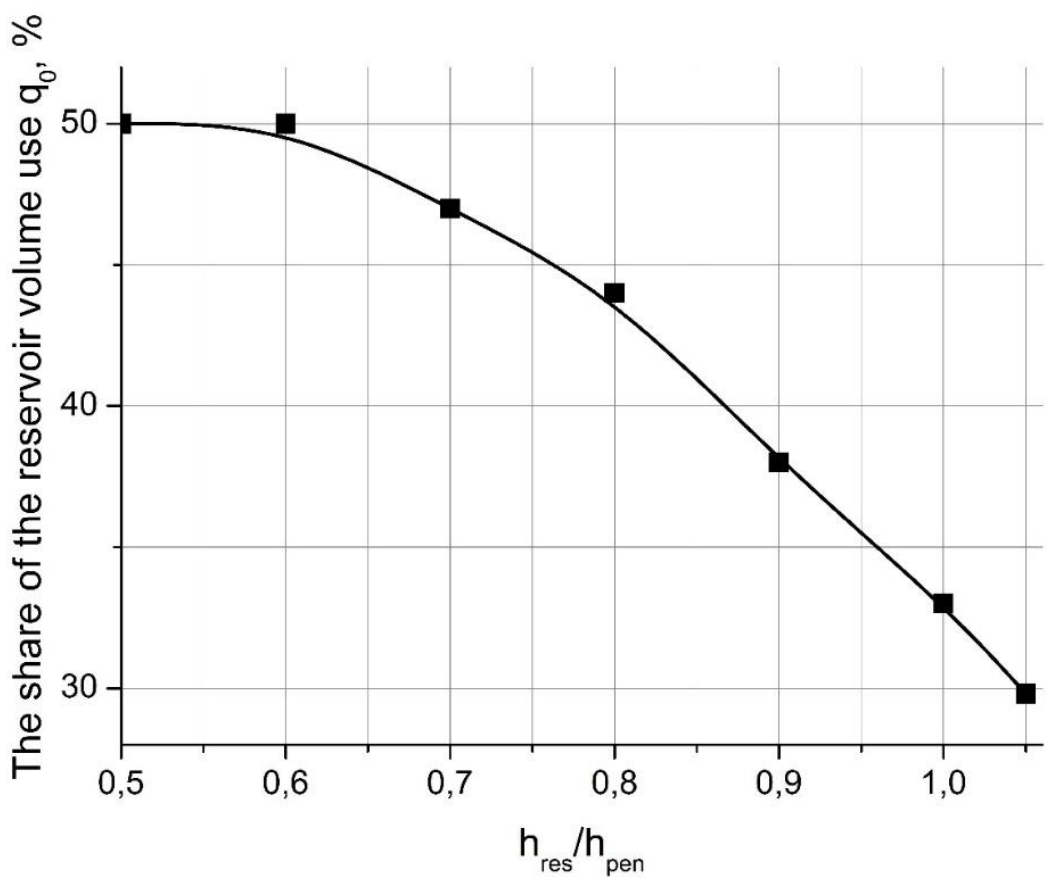

Fig.2. Graph of dependence of the share of the reservoir volume use on the ratio of the height of the water layer and the penetration depth

Based on the results of the experiments, it can be concluded that for enterprises with a small amount of wastewater, where large areas of treatment facilities are not required, it is possible to carry out aeration in shallow reservoirs (the depth of the reservoir is $(0.6 \ldots$ $0.7) \mathrm{h}_{\text {pen }}$ ). Table 3 shows the relationship between the spray pattern diameter and the jet penetration depth.

Table 3. Relationship between the diameter of the gas-liquid plume $d_{p l}$ and the jet penetration depth $h_{p e n}$

\begin{tabular}{|l|l|l|l|l|l|}
\hline $\mathrm{h}_{\text {res }} / \mathrm{h}_{\text {pen }}$ & 1.05 & 1 & 0.7 & 0.6 & 0.5 \\
\hline $\mathrm{d}_{\text {pl }} / \mathrm{h}_{\text {pen }}$ & 0.35 & 0.35 & 0.438 & 0.7 & 0.9 \\
\hline
\end{tabular}

From Figure 2 and Table 3 show that the most significant relative diameter of the spray pattern and the maximum utilization of the reservoir volume are at the ratio of $h_{\text {res }} / h_{\text {pen }}=0.5 \ldots 0.7$. Consequently, for effective aeration of waste fluid in small facilities, it is necessary to select the depth of the reservoir so that the specified ratio is observed since, in this case, the gas-liquid jet breaks against the bottom of the reservoir and expands the aerated zone.

The obtained data can be used in calculating the aerator with a certain liquid flow rate $\mathrm{Qm}^{3} /$ day, at given biological oxygen demand (BOD) in incoming water $L_{0}$ and purified water $L_{t}, \mathrm{mg} / \mathrm{l}$. The calculation is based on the determination of the oxygen transfer coefficient provided by the design of this aerator and in comparing this coefficient with the required $K_{L a}(20)$ in terms of the discharged liquid. Therefore, for the calculation, it is necessary to have data on the dependencies of the jet penetration depth $h_{p e n}$ and the oxygen transfer coefficient on the liquid flow rate.

Based on the results of our own research, as well as using the data of Shukla [22] using modeling in the Origin and Excel packages, an equation was obtained for the dependence of the required jet velocity on the depth of its penetration: 


$$
v=0,0211\left(\frac{h_{p e n}^{5}}{n^{0.775 \cdot P^{1.8}}}\right)^{1 / 2}
$$

Where $\mathrm{n}$ is the number of holes in the nozzle, $\mathrm{P}$ is the total perimeter of holes, $\mathrm{mm}$

Figures 3 and 4 show graphs of these dependencies for nozzles with a different number of "keyway" holes of a total area of $595 \mathrm{~mm}^{2}$.

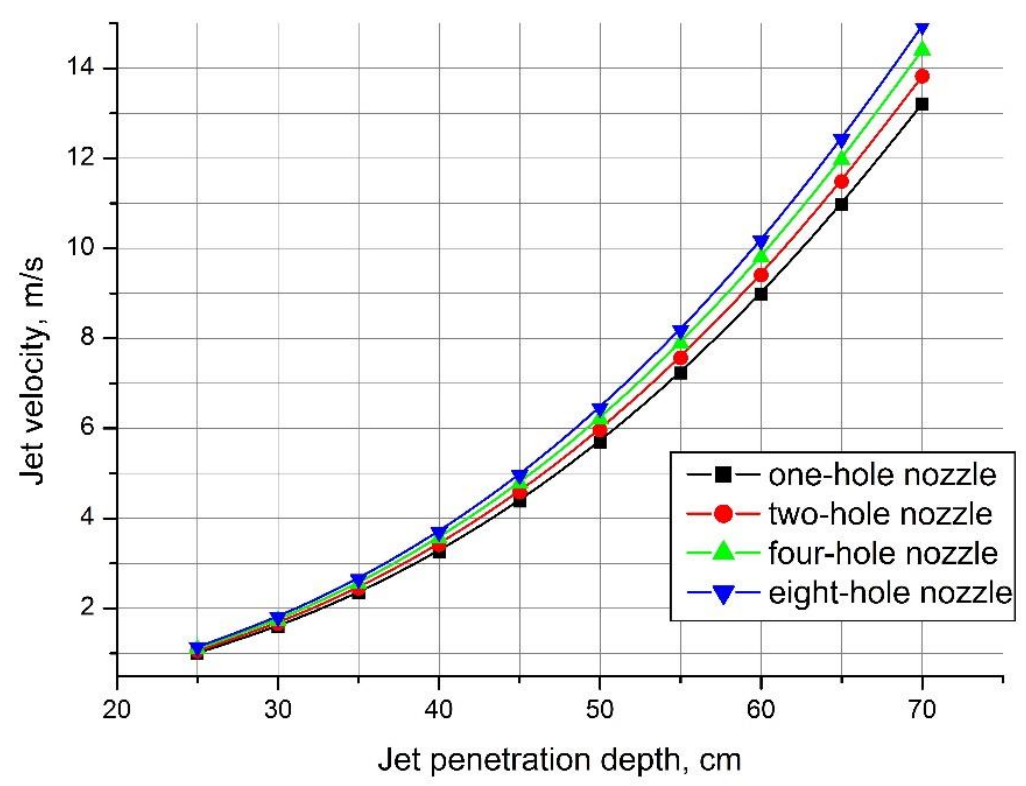

Fig.3. Graph of dependence of the jet penetration depth $\mathrm{h}_{\text {pen }}$ on jet velocity

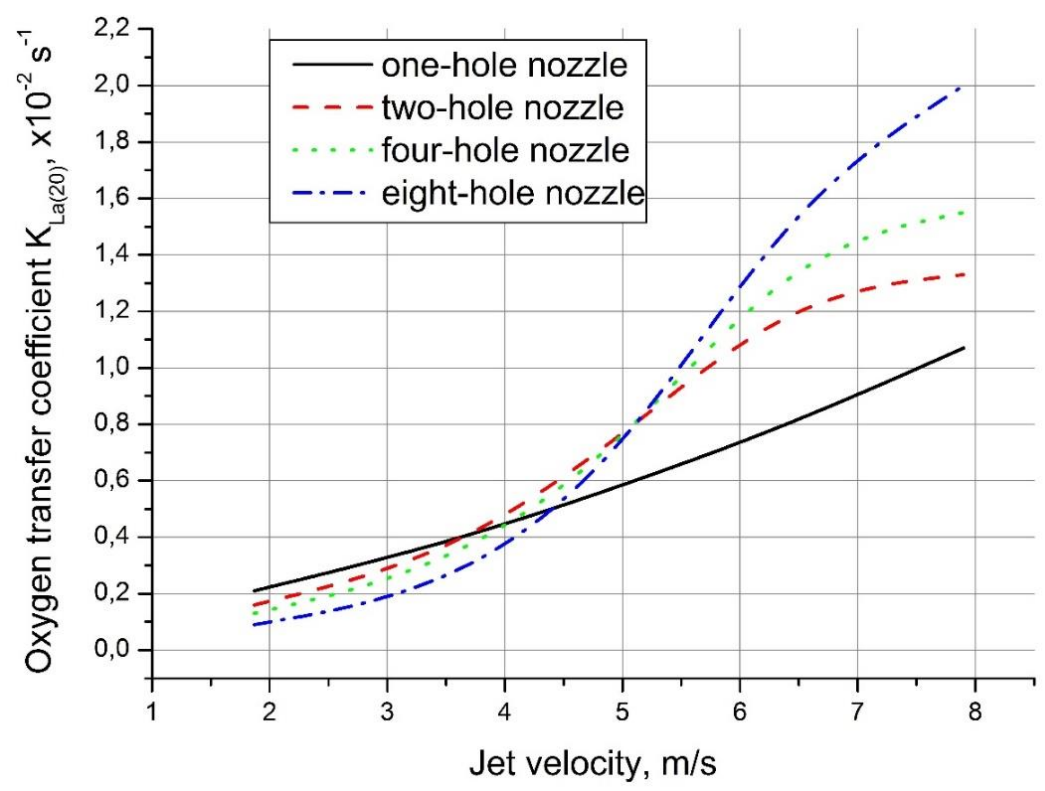

Fig. 4. Graph of dependence of the oxygen transfer coefficient on jet velocity Based on the data obtained, the following algorithm for calculating the jet aeration plant is proposed. 
The procedure for calculating the jet aerator

Initial data: waste water consumption liquid $\mathrm{Q}_{\mathrm{ww}}, \mathrm{m}^{3} /$ day; BOD of incoming wastewater $\mathrm{L}_{0}, \mathrm{mg} / \mathrm{l}$; required BOD of purified water $\mathrm{L}_{\mathrm{t}}, \mathrm{mg} / \mathrm{l}$.

1. The number $k$ of aerators with holes of the "keyway" type of a certain type is accepted (table 1)

2. The jet penetration depth is set, $h_{p e n}, \mathrm{~cm}$

3. The reservoir depth is set, $h_{\text {res }}=0.7 h_{\text {pen }}, \mathrm{cm}$

4. Gas-liquid plume diameter is $d_{p l}=0.438 h_{p e n}$ (Table 2)

5. Gas-liquid plume cross-sectional area $F=\frac{\pi d_{p l}^{2}}{4}, \mathrm{~m}^{2}$

6. The required surface area of the reservoir $F_{\text {res }}=k \cdot F, \mathrm{~m}^{2}$

7. Jet velocity $v$ in the nozzle holes (formula (4), Fig. 3), $\mathrm{m} / \mathrm{s}$

8. The required water flow rate through one nozzle, $\mathrm{q}_{1}: q_{1}=v \cdot S, \mathrm{~m}^{3} / \mathrm{s}$

9. The flow rate through $k$ nozzles $Q=q_{1} \cdot k, \mathrm{~m}^{3} / \mathrm{s}$

10. Standard volumetric mass transfer coefficient $\mathrm{K}_{\mathrm{La}(20)}$ is determined depending on $\mathrm{q}_{1}$ (see graph in Figure 4)

11. $\mathrm{K}_{\mathrm{La}(20)}$ check

a) the required amount of $\mathrm{O}_{2}$

$$
G=K_{L a}\left(C_{p}-C\right) \cdot W
$$

where $C_{s}$ is the solubility of oxygen in water $\left(C_{s}=9.1 \mathrm{mg} / 1\right.$ at $\left.20^{\circ} \mathrm{C}[26]\right)$,

$\mathrm{C}$ is the average oxygen concentration in the treatment facility $\mathrm{C}=2 \mathrm{mg} / \mathrm{l}$,

$\mathrm{W}$ is the volume of the facility, $\mathrm{m}^{3}$

b) on the other hand, the required amount of $\mathrm{O}_{2}$ :

$$
G=z\left(L_{0}-L_{t}\right) Q_{w w}
$$

$\mathrm{z}$ is the specific consumption of $\mathrm{O}_{2}$ of the removed $\mathrm{BOD}_{\text {total }}, \mathrm{mg} / \mathrm{mg}(\mathrm{z}=1.25$ [26]),

$\mathrm{Q}_{\mathrm{ww}}$ is the rate of waste liquid, 1/s (taken according to the initial data).

c) required value of $\mathrm{K}_{\mathrm{La}(20)}$

$$
K_{L a(20)}=\frac{z\left(l_{0}-L_{t}\right) Q_{C T}}{\left(c_{P}-e\right) V} \geq K_{L a(20)^{\prime}}
$$

$K_{L a(20)}{ }^{\prime}$ is the value defined in item 10 .

If the condition $\left.K_{\mathrm{La}(20)} \geq K_{\mathrm{La}(20)}\right)^{\prime}$ is not met, recalculation of the aerator is required using other options of the initial data $\left(\mathrm{k}, \mathrm{h}_{\mathrm{pen}}\right)$.

12. Aeration time is $\tau=\frac{V}{Q_{C T}}, \mathrm{~s}$

13. Pump selection is done by $Q$ value (item 9)

\section{Conclusions}

The studies' results show that it is advisable to use jet aeration for biological treatment in small facilities. Experimental verification for a jet aerator with "keyway" holes showed that the sizes of the actively aerated zone depend on the ratio of the possible depth of the reservoir (the water layer thickness) and the possible penetration depth of the gas-liquid jet. 
At that, it was determined that limiting the depth of the reservoir allows increasing the size of the aerated zone and the degree of utilization of the reservoir volume. With a decrease in the relative depth of the reservoir from 1.05 to 0.5 (2.1 times), the share of the beneficial use of the reservoir volume increases from $29.8 \%$ to $50 \%$ (1.68 times).

The technique developed based on experimental data allows for a simple calculation of the jet aerator, which can accelerate the design processes of small treatment facilities.

\section{Reference}

1. Shapovalova L, Nurmatova V, Kirshina E, Smolkova O, Improved wastewater purification technology for villages in countryside of the Republic of Uzbekistan Proc. of the 19th Int. Conf. Sakharov readings 2019: environmental problems of the XXI century (Minsk) vol 3 (Minsk: ITC of the Ministry of Finance) pp 106-109. (2019)

2. Muminova $\mathrm{R}$ and Kazimova $\mathrm{N}$, The problem of wastewater treatment in Uzbekistan $\mathrm{J}$. Young scientist 22 (102) pp 47-48, (2015)

3. Serpokrylov N, Smolyanichenko A and Lesnikov I, Comparative analysis of aerators for wastewater purification and unified aeration criteria J. SGASU Bulletin. Urban planning and architecture 2 pp 97-100. (2011)

4. Gogina E and Makisha N, Reconstruction of wastewater treatment plants in Russia, approaches and solutions. J. Applied Mechanics and Materials vol 361-363 pp 628631 (2013)

5. Itano T, Inagaki C, Hashimoto R, Negoro N, Hyodo J and Honda S. Water circulation induced by mechanical aerators in a rectangular vessel for shrimp aquaculture J. Aquacultural Eng. 85 pp 106-113 (2019).

6. Xiangju C, Jun X, Deguang Y and Yingxue Z, Calculated analysis of oxygen transfer from air bubble-water interface and turbulent water surface in microporous aeration systems. Transactions of the Chin. Society of Agric. Eng. (29) 13 pp 190-199. (2012)

7. Abdukodirova $\mathrm{M}$ and Radkevich M, Opportunities for application of jet aerators in biological cleaning facilities J. Universum: Techn.Sci. 5 (74) Part 1 pp 60-63, (2020)

8. Orekhov G, Flow characteristics and peculiarities of cavitation phenomena in counter-vortex flow energy dissipators of hydraulic structures. The Eurasian Scientific Journal 3 (11), (2019)

9. Samokhvalova A, Kuksova A and Yurchenko V, The main types of jet aerators used in wastewater treatment $J$. Naukoviy visnik budivnistva (Scient. Bul. of the Buil.) 3 pp 124-128. (2015).

10. Lobov W, Sugak A and Gontscharov G, Die Bewegungstraiektorie einer Gasblase im stark durchlüfteten Bereich von Strahlapparaten mit unterschiedlich geformten Düsen. Technische Fachhochschule Wildau. Wissenschaftliche Beiträge 1 pp 159-162 (2000)

11. Cummings $\mathbf{P}$ and Chanson $\mathrm{H}$. Air entrainment in the developing flow region of plunging jets - Part 1: Theoretical Development J. of Fluids Eng. 119 pp. 597-602. (1997)

12. Bouvard J, B'eguin C, Etienne S, Scott D and Bornard L, Characterization of bubble cloud quality and evolution using optical probes. Proc. of the 29th IAHR Symposium on Hydraulic Machinery and Systems IOP Conf. Ser.: Earth Environ. Sci. 240 062051 (2019).

13. Lima Neto I E, Zhu D Z and Rajaratnam N, Air Injection in Water with Different Nozzles. J. Environ. Eng. 4 (134) pp 283-294. (2008).

14. Lima D D and Lima Neto I E, Effect of nozzle design on bubbly jet entrainment and oxygen transfer efficiency J. Hydr. Eng. 8. (144). (2018) 
15. Ledyan Yu P, Selivonchik B B and Shcherbakova M K, The use of jet aeration to improve the efficiency of water purification in the recirculating water supply system of the foundry Casting and metallurgy 4 (49) pp 161-164. (2008).

16. Ledyan Yu P and Selivonchik B B Application of jet aeration to intensify the flotation process. Bulletin of BNTU 6 pp 52-55. (2008)

17. Inventor's certificate 798051 (USSR). Method of liquid aeration (N.F. Meshcheryakov, Yu.V. Ryabov, M.A. Podvigin, M.A.Gamilov). Publ. in BI, (3) (1981)

18. Kusabiraki D, Niki H, Yamagiwa K and Ohkawa A, Gas entrainment rate and flow pattern of vertikal plunging liguid jets The Can. J. Chem. Eng. 68 (6) pp 893 - 903, (1990).

19. Van de Sande E and Smith Y.M, Set break-up and air entrainment by low velocity turbulent water jets Chem. Eng. Sci. 31. pp 219-224. (1976).

20. Safronova E V, Spiridonov A V and Mitinov A V, Design and calculation of effective jet devices for the "liquid-gas" system Bull. of Polotsk State University 6 pp 190-194, (2016).

21. Abaev G N and Chernyavskaya E V, Patterns of hydrodynamics and mass transfer in jet device. Eng. Phys. J. 74 (3) pp $184-188$. (2001).

22. Shukla B K and Goel A, Study on oxygen transfer by solid jet aerator with multiple openings. Eng. Sci. and Techn., an Int. J. 21 pp 255-260. (2018).

23. Suciu $\mathrm{G}$ and Smigelschi $\mathrm{O}$, Size of submerged biphase region in plunging jet systems Chem. Eng. Sci. 31 (12) pp 217- 220. (1976).

24. McKeogh E Y and Ervine D A, Air entrainment rate and diffusion pattern of plunging liquid jets Chem. Eng. Sci. 36 (7) pp 1161-72. (1981).

25. Roy A. K., Maiti B. and Das P.K. Visualization of Air Entrainment by a Plunging Jet. 2013 Procedia Engineering 56, pp 468-473. DOI: 10.1016/j.proeng.2013.03.148. (2013).

26. Guidelines for determining the need for electrical energy for technological needs in the field of water supply, sewerage and wastewater treatment 2007, (Moscow: Center for Municipal Economy and Law) p 16. (2007). 